\title{
POLR1B is upregulated and promotes cell proliferation in non-small cell lung cancer
}

\author{
FAN YANG, HAITAO LIU, JUNJIE ZHAO, XINGJIE MA and WEIBO QI \\ Department of Cardiothoracic Surgery, The First Affiliated Hospital of Jiaxing University, \\ Jiaxing, Zhejiang 314001, P.R. China
}

Received April 15, 2019; Accepted September 11, 2019

DOI: $10.3892 / \mathrm{ol} .2019 .11136$

\begin{abstract}
The aim of the present study was to investigate the functions of RNA polymerase I subunit B (POLR1B) in lung cancer. Reverse transcription-quantitative PCR was used to measure the mRNA expression level of POLR1B in human lung cancer cell lines including A549, NCI-H1299, NCI-H1975 and NCI-H460. A lentivirus vector transfection system was used to knockdown POLR1B in A549 cells. The Celigo Cell Counting method, MTT and colony formation assays were used to investigate the proliferation of knocking-down POLR1B in A549 cells. Flow cytometry assay was used to investigate apoptosis rates. Co-expression analysis and microarray analysis were used to identify POLR1B targets in NSCLC. The Celigo Cell Counting method, MTT and colony formation assay revealed that the proliferation rates of lung cancer cells were significantly suppressed when POLR1B was silenced by lentivirus-mediated RNA interference. In addition, knocking-down the expression of POLR1B induced lung cancer cell apoptosis, visualized via flow cytometry. Bioinformatics revealed that POLR1B regulated multiple biological processes in NSCLC, including positive regulation of glucose import, and autophagosome assembly. The present study also identified several key targets of POLR1B, including ADRA1D, NR4A1, MYC, BOP1, DKC1, RRP12, IPO4, MTHFD2, CTPS1, GARS and NOC2L. The data from the present study suggest that POLR1B is an important modulator of lung cancer cell proliferation and indicate that POLR1B
\end{abstract}

Correspondence to: Dr Weibo Qi, Department of Cardiothoracic Surgery, The First Affiliated Hospital of Jiaxing University, 1882 Zhonghuan South Road, Jiaxing, Zhejiang 314001, P.R. China E-mail: qiweibo0221@163.com

Abbreviations: POLR1B, RNA polymerase I subunit B; RNAi, RNA interference; NSCLC, non-small cell lung cancer; CTLA4, $\mathrm{T}$ lymphocyte antigen 4; PD-1, programmed cell death 1; FBS, fetal bovine serum; RT-qPCR, quantitative reverse transcription polymerase chain reaction

Key words: polymerase I subunit B, lung cancer, proliferation may be further selected as a potential anticancer therapeutic target for human lung cancer.

\section{Introduction}

Lung cancer is the leading cause of cancer-associated mortality worldwide in 2011 (1), and $~ 80 \%$ of all cases of lung cancer are non-small cell lung cancer (NSCLC) (2). However, there are currently a limited number of effective approaches for preventing the development of NSCLC, and improving the survival rates of those patients with the disease (3). The standard treatment for the majority of patients with advanced NSCLC is platinum-based chemotherapy followed by second-line cytotoxic chemotherapy (4). Nevertheless, the overall survival time of patients receiving this treatment for advanced NSCLC remains poor, with a median time of $\sim 12$ months (3).

Novel immunotherapeutic approaches have been developed to treat NSCLC, including chimeric antigen receptor T cells (CAR-T) and monoclonal antibodies that block immune checkpoint molecules, such as programmed cell death 1 (PD-1) and cytotoxic T lymphocyte antigen 4 (CTLA4) (5-9). However, the efficiency of CAR-T treatment for solid tumors (including NSCLC) requires improvement, and the potential drawback of anti-CTLA4 and anti-PD1 therapy is their immune-potentiating side effects (10-12). Therefore, the identification of novel prognostic markers or molecular targets for the effective treatment of NSCLS is paramount.

RNA polymerase I (pol I), a multi-subunit enzyme consisting of $>6$ polypeptides, is essential for the transcription of ribosomal RNA (rRNA) and the production of the primary components of ribosomes $(13,14)$. In yeast, the majority of the pol I complex comprises the two largest subunits, Rpa1 and Rpa2; RNA polymerase I subunit RPA2 (POLR1B) is the human Rpa2 homologue $(15,16)$. Previous studies have revealed that an increase in rRNA synthesis is associated with poor prognosis in patients with multiple types of cancer, such as lymphoma and rhabdomyosarcoma (17-19). These reports indicated the potential regulatory roles of POLR1B in human cancer. However, the roles of POLR1B in lung cancer have not yet been identified; thus, there is accumulating interest in investigating the function of POLR1B in cancer cell proliferation. In the present study, the functions of POLR1B in lung cancer were investigated, and the molecular mechanisms that regulate lung cancer cell proliferation were further elucidated. 


\section{Materials and methods}

Public datasets analysis. Gene Expression Profiling Interactive Analysis (GEPIA) is a web server for gene expression profiling and interactive analyses in human cancer (20). It provides several key interactive customization features, such as differential expression analysis, patient survival analysis and similarity gene detection. The present study analyzed the expression profile of POLR1B in different types of human cancer using GEPIA datasets. And the association between POLR1B and several key NSCLC regulators, including tumor protein (TP)53, epidermal growth factor receptor (EGFR), and the GTPases KRas (KRAS) and NRas (NRAS), was also assessed using GEPIA datasets.

Co-expression analysis of POLR1B in NSCLC was then performed using the cBioPortal database (21). Furthermore, GSE18842 (22) was employed to evaluate the expression levels of POLR1B in NSCLC and normal samples. A total of 46 non-small cell lung cancer samples and 45 normal lung tissues were included in this dataset.

Cell lines and cell culture. The human 293T cell line and lung cancer cell lines A549, NCI-H1299, NCI-H1975 and NCI-H460 were purchased from the Cell Bank of the Type Culture Collection. The A549 cells were maintained in F-12K medium (Invitrogen; Thermo Fisher Scientific, Inc.) and the NCI-H1299, NCI-H1975 and NCI-H460 cells were cultured in RPMI-1640 medium (Gibco; Thermo Fisher Scientific, Inc.). The human 293T cells were maintained in DMEM high glucose medium (Gibco; Thermo Fisher Scientific, Inc.). All media were supplemented with $10 \%$ fetal bovine serum (FBS), $100 \mathrm{U} / \mathrm{ml}$ streptomycin and penicillin (all from Gibco; Thermo Fisher Scientific, Inc.). The present study detected the expression levels of POLR1B in four NSCLC cell lines. The results revealed that POLR1B was highly expressed in all NSCLC cell lines. Despite the expression of POLR1B in NCI-H1975 cells being higher than other cell lines, however, the differences were not significant. A549 cell line was one of the most widely used NSCLC cell line in lung cancer biology research and selected for further study.

Construction of lentiviruses for POLRIB knockdown. The target DNA sequence of POLR1B (5'-GACCATTGGGAT GTTAATT-3') was designed by Shanghai GeneChem Co. Ltd. According to the target sequence of POLR1B, two single chain DNA sequences (shRNA S1 and shRNA S2) were designed: shRNAS1, 5'-CCGGATGACCATTGGGATGTTAATTCT CGAGAATTA ACATCCCAATGGTCTTTTTTG-3'; and shRNA S2, 5'-AATTCAAAAAATGACCATTGGGATGTT AATTCTCGAGAATTAACATCCCAATGGTCAT-3'. The shRNAs were annealed and ligated into the linearized GV115 lentiviral vector, and the resulting plasmid was used to transform DH5 $\alpha$ competent cells (Shanghai Genechem Co., Ltd., Shanghai, China). The plasmid was extracted from the DH5 $\alpha$ cells and verified by restriction endonuclease digestion followed by Sanger sequencing. Lentiviral vector DNA and packaging vectors $(1 \mu \mathrm{g} / \mathrm{ml}$; pHelper 1.0 and pHelper 2.0; Shanghai Genechem Co., Ltd.) were then transfected into 293T cells (Sangon Biotech, Co., Ltd.) using Lipofectamine ${ }^{\circledR}$ 2000 (Invitrogen; Thermo Fisher Scientific, Inc.). The empty vector of GV115 lentiviral vector (Shanghai Genechem Co., Ltd.) was used as the shRNA control. After $48 \mathrm{~h}$ of culture, supernatants containing lentiviruses, including shPOLR1B and shRNA control were harvested, respectively. Purification was then performed using ultracentrifugation at $1,000 \mathrm{x} g$ and $4^{\circ} \mathrm{C}$ for 2 min (Himac CT15RE; Hitachi) and the titer of lentivirus was determined. A549 lung adenocarcinoma cells were infected with the shPOLR1B lentivirus, and cells in the control group were infected with a lentivirus carrying an empty vector. Cells were cultured in RPMI-1640 medium with lentiviruses at a multiplicity of infection of 10 for $24 \mathrm{~h}$ at $37^{\circ} \mathrm{C}$. Fresh culture medium was then used to replace the old media. Fluorescence was measured $72 \mathrm{~h}$ post-infection when the achieved infection efficiency was $80 \%$, and the expression levels of POLR1B were analyzed using reverse transcription-quantitative (RT-q)PCR and western blotting. The fluorescence and infection efficiency were determined using an inverted fluorescence microscope (magnification, x200; IX-71; Olympus).

Cell viability assay. The cell viability was determined using an MTT assay (Sigma-Aldrich; Merck KGaA). Briefly, A549 cells infected with shPOLR1B-lentivirus or the corresponding negative control were seeded into 96 -well plates $\left(2 \times 10^{3}\right.$ cells $/$ well $)$, and incubated at $37^{\circ} \mathrm{C}$ for 5 days. Following incubation, $20 \mu 1$ MTT solution $(1 \mathrm{mg} / \mathrm{ml})$ was added to each well and incubated for a further $4 \mathrm{~h}$ at $37^{\circ} \mathrm{C}$ at each time points, including days 1,2,3,4 and 5. The MTT solution was then aspirated and $100 \mu \mathrm{l}$ DMSO (Sigma-Aldrich; Merck KGaA) was added to dissolve the formazan crystals. The number of viable cells was counted using an automated microplate reader (Molecular Devices, LLC) at a wavelength of $570 \mathrm{~nm}$.

Cell proliferation analysis with the Celigo ${ }^{\circledR}$ adherent cell cytometry system. Briefly, A549 cells infected with shPOLR1B-lentivirus or the shRNA-lentivirus control were trypsinized in the logarithmic growth phase using trypsin (Gibco; Thermo Fisher Scientific, Inc.), resuspended in standard medium and seeded into 96 -well plates $\left(2 \times 10^{3}\right.$ cells/well). After plating, Celigo ${ }^{\circledR}$ Image Cytometer (Nexcelom) was used to evaluate the number of cells by scanning green fluorescence daily for 5 days at room temperature as previously described (23).

Colony forming assays. A549 cells were cultured in F-12K complete media (10\% FBS, $1 \%$ penicillin/streptomycin) and $1 \times 10^{3}$ cells/well were seeded into 6 -well plates; the medium was replaced every 3 days. After culturing for 10 days to form colonies, each well was washed with $1 \mathrm{ml}$ PBS and the cells were fixed with $1 \mathrm{ml} \mathrm{4 \%}$ paraformaldehyde (Beyotime Institute of Biotechnology) for $15 \mathrm{~min}$ at room temperature. The cells were then washed with PBS and $500 \mu$ l Giemsa stain (Sigma-Aldrich; Merck KGaA) was added to each well and incubated for 15-20 min at room temperature. Excess staining solution was washed off with PBS and the colonies were observed with an inverted light microscope (x200 magnification; IX71; Olympus, Tokyo, Japan) and counted using Image J software (version 4.0; National Institutes of Health).

Apoptosis analysis. Apoptosis analysis was performed using an Annexin-V/FITC apoptosis detection kit (Invitrogen; Thermo Fisher Scientific, Inc.) according to the manufacturer's protocol. 
Briefly, cells were seeded into T25 flasks and cultured at $37^{\circ} \mathrm{C}$ for $48 \mathrm{~h}$. When the cell density had reached $\sim 80 \%$, the cells were collected and resuspended in ice-cold PBS, prior to fixing with pre-cooled $75 \%$ ethanol for $30 \mathrm{~min}$ at $4^{\circ} \mathrm{C}$. After $30 \mathrm{~min}$, the cells were centrifuged at $100 \mathrm{x} \mathrm{g}$ and washed twice with PBS. The pellets were then resuspended in annexin-V/FITC and propidium iodide (Invitrogen; Thermo Fisher Scientific, Inc.), and incubated at room temperature for $15 \mathrm{~min}$ in the dark. The cells were washed twice with PBS and analyzed using a FACS Calibur flow cytometer (BD Biosciences). Cell apoptosis data was analyzed using FCS Express software (version 3.0; DeNovo).

RNA extraction and RT-qPCR analysis. The A549, NCI-H1299, NCI-H1975 and NCI-H460 cells were lysed using TRIzol ${ }^{\circledR}$ reagent (Gibco; Thermo Fisher Scientific, Inc.) and the total RNA was isolated. cDNA was generated using a cDNA synthesis kit (Takara Biotechnology Co., Ltd.,) with $2 \mu \mathrm{g}$ RNA, according to the manufacturer's instructions. The mRNA expression levels were determined using SYBR ${ }^{\circledR}$ Green assays and the Applied BioSystems 7300 detection system; the SYBR ${ }^{\circledR}$ Green qPCR SuperMix-UDG was purchased from Invitrogen; Thermo Fisher Scientific, Inc. The relative gene expression levels were calculated using the $2^{-\Delta \Delta \mathrm{Cq}}$ method (24), and the following primers were used for qPCR: POLR1B: Forward, 5'-TCCGAATGTTGATTATGCCTC G-3', and reverse, 5'-TGACAGCGGAATGTTCTTCCC-3'; GAPDH: Forward, 5'-TGACTTCAACAGCGACACCCA-3', and reverse, 5'-CACCCTGTTGCTGTAGCCAAA-3'. The qPCR reaction conditions were as follows: Initial denaturation $10 \mathrm{~min}$ at $95^{\circ} \mathrm{C}$; denaturation $10 \mathrm{sec}$ at $95^{\circ} \mathrm{C}$; annealing $20 \mathrm{sec}$ at $60^{\circ} \mathrm{C}$; and extension $15 \mathrm{sec}$ at $72^{\circ} \mathrm{C}$; for 40 cycles.

Western blot analysis. The proteins were extracted from A549 cells using ice-cold radioimmunoprecipitation assay lysis buffer (Beyotime Institute of Biotechnology). The concentration of protein was determined using a BCA kit (Thermo Fisher Scientific, Inc.). A total of $40 \mu \mathrm{g}$ solution was loaded per lane onto SDS-PAGE for electrophoresis separation $(10 \%$ separation gel and 5\% spacer gel), and then transferred to polyvinylidene fluoride (PVDF) membranes. Subsequently, the membranes were blocked in $5 \%$ bovine serum albumin (Gibco; Thermo Fisher Scientific, Inc.) at room temperature for $1 \mathrm{~h}$ then probed with the primary antibodies overnight at $4^{\circ} \mathrm{C}$, including anti-POLR1B (cat. no. ab101977, 1:1,000) and anti-GAPDH (cat. no. ab181602, 1:1,000) (both from Abcam). The next day, the membranes were washed three times using $0.1 \%$ tris-buffered saline and Tween 20 (TBST; Sigma-Aldrich; Merck KGaA), 5 min each time, then horseradish peroxidase (HRP)-conjugated anti-rabbit (cat. no. IH-0011, 1:5,000; Dingguo Changsheng Biotechnology, Beijing, China) and horseradish peroxidase (HRP)-conjugated anti-mouse antibodies (cat. no. IH-0031, 1:5,000; Dingguo Changsheng Biotechnology) were added and incubated for $1 \mathrm{~h}$ at room temperature, then washed with $0.1 \%$ TBST three times. The protein bands were visualized using an enhanced chemiluminescence assay kit (Beyotime Institute of Biotechnology) and the Luminescent image analyzer (GE Healthcare). The intensity of the bands was quantified using Image $\mathrm{Lab}^{\mathrm{TM}}$ Software (version 4.1; Bio-Rad Laboratories, Inc.).
Microarray and expression datasets. Total RNA was extracted using TRIzol ${ }^{\circledR}$ reagent and was quantified by the NanoDrop ND-2000 (Thermo Fisher Scientific, Inc.). Global expression of mRNAs in 3 control A549 samples and 3 POLR1B knockdown A549 samples were examined using the GeneChip PrimeView Human Gene Expression Array (Thermo Fisher Scientific, Inc.). The raw data of the mRNA expression profiles were analyzed using the limma model (11) on R/Bioconductor software version 2.15.1 (www.bioconductor.org). Background correction, quartile data normalization and probe summarization were applied for the original data. The limma method in Bioconductor (http://www.bioconductor.org) was used to identify the genes that were differentially expressed between the two groups. Genes with an adjusted $\mathrm{P}<0.05$ after FDR correction and a fold change of $>2$ or $<0.5$ were considered as differentially expressed genes.

Construction of protein-protein interaction (PPI) network. The present study used the Search Tool for the Retrieval of Interacting Genes/Proteins (STRING; version 11.0; http://string-db.org) (25) to construct the PPI network. Cytoscape software (version 3.70; www.cytoscape.org) is a common open-source software tool that is useful in the visual evaluation of biomolecule interaction networks comprising of protein, gene and other forms of interactions (26).

Statistical analysis. All experiments were performed in triplicate. Statistical analysis was performed by SPSS software (verison 18.0; SPSS, Inc.). The results are presented as the mean \pm standard error of the mean. Statistical significance between the shRNA-transfected cell groups was determined using Student's t-test. The median expression (7.13) of POLR1B in all human cancer samples were considered as the cut-off to classify groups into POLR1B-high and POLR1B-low expression. A Kaplan-Meier survival curve was created and compared with the log-rank test results. For $>2$ groups, one-way analysis of variance followed by Newman-Keuls post hoc test was used. $\mathrm{P}<0.05$ was considered to indicate a statistically significant difference.

\section{Results}

POLRIB is upregulated in human cancer, particularly NSCLC. In order to investigate the expression profile of POLR1B in different types of human cancer, gene expression profiling interactive analysis (GEPIA) datasets were analyzed. As presented in Fig. 1A, the expression levels of POLR1B were demonstrated to be upregulated in breast invasive carcinoma, colon adenocarcinoma, diffuse large B-cell lymphoma, low-grade glioma, lung adenocarcinoma (LUAD), lung squamous cell carcinoma (LUSC), ovarian cancer, pancreas adenocarcinoma, rectum adenocarcinoma, skin cutaneous melanoma, stomach adenocarcinoma and thymoma, compared with normal sample tissues. The median expression of POLR1B in all human cancer samples were considered as the cut-off to classify groups into POLR1B-high and POLR1B-low expression. Kaplan-Meier survival analysis revealed that a higher POLR1B expression level was associated with significantly shorter overall survival times in 4,750 patients with various types of cancer (Fig. 1B). These results suggested that POLR1B may act as an oncogene in human cancer. 
A

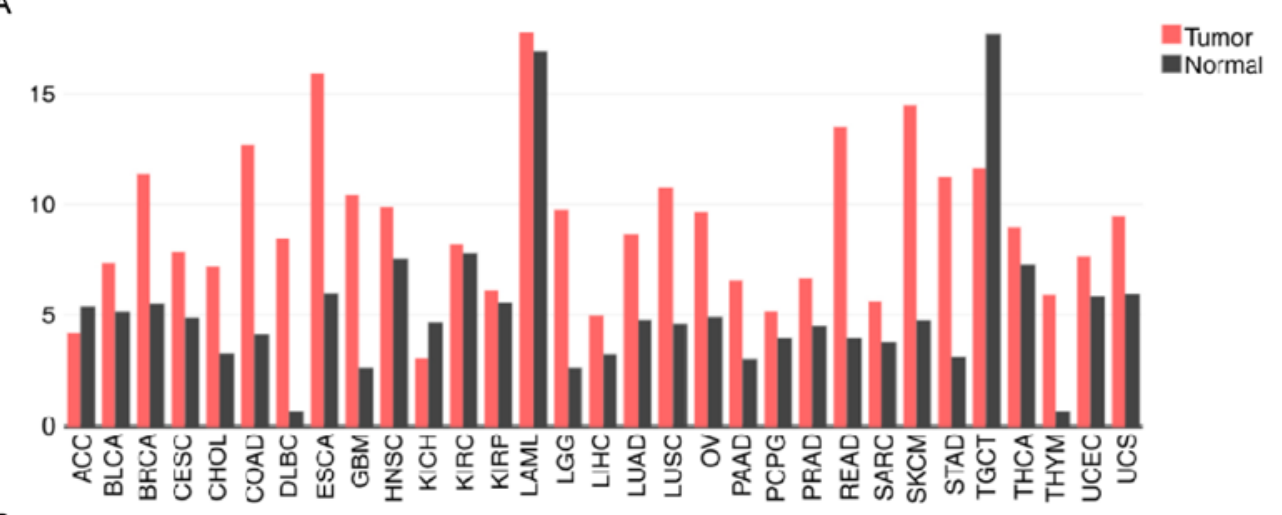

B

C
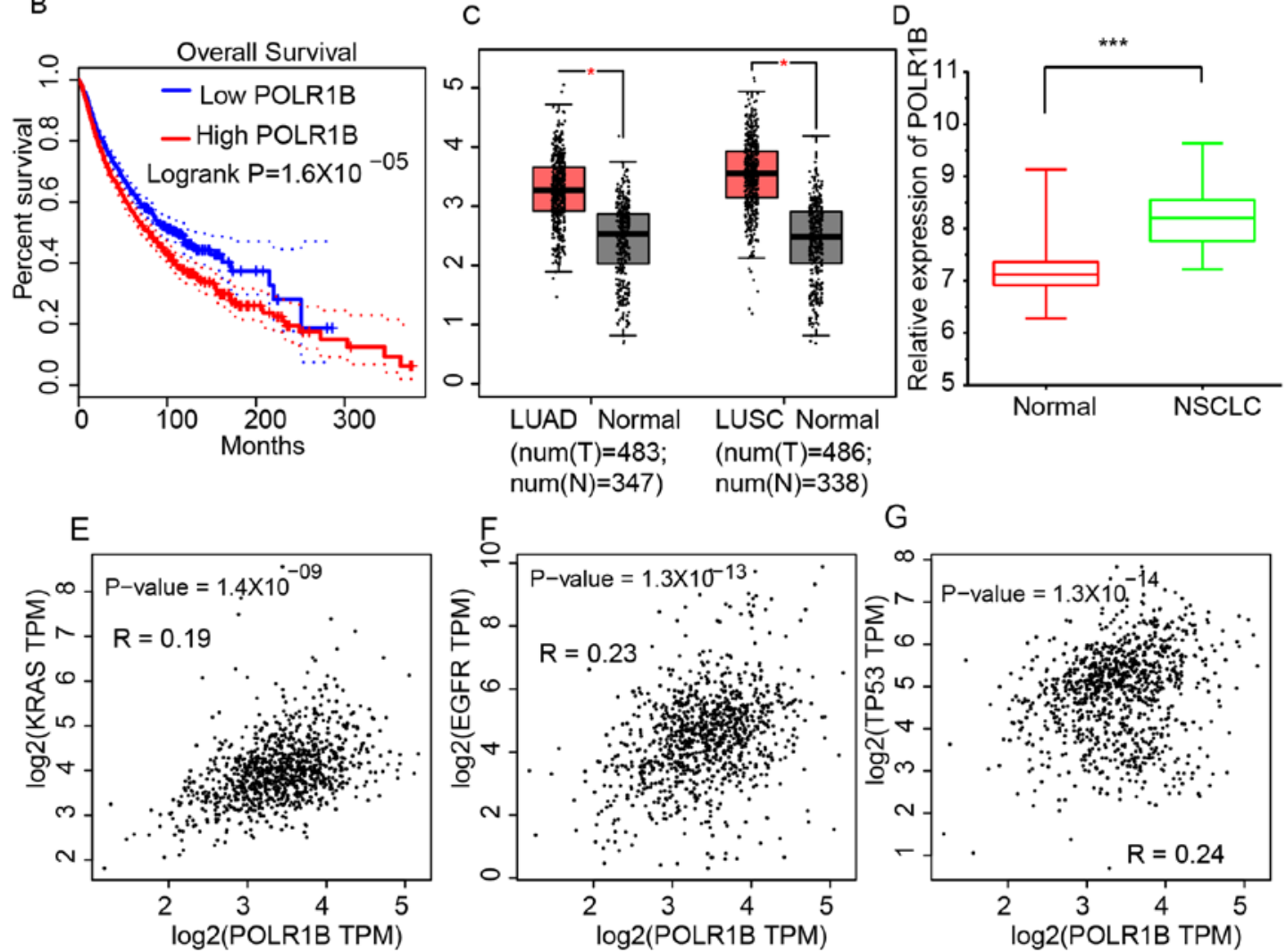

G

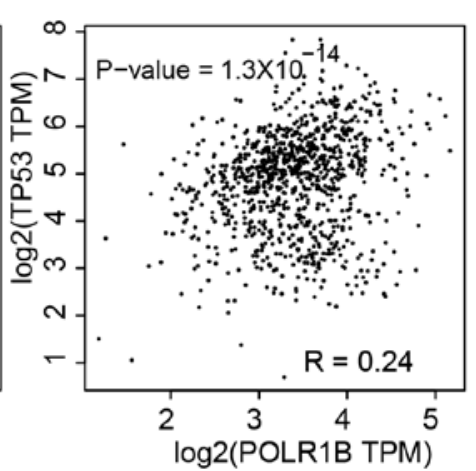

Figure 1. POLR1B is upregulated in NSCLC samples. (A) The expression pattern of POLR1B in human cancers was analyzed using gene expression profiling interactive analysis datasets. (B) The higher expression of POLR1B was associated with shorter overall survival time in human cancer. (C) POLR1B was upregulated in LUAD and LUSC samples compared with normal tissues. Red bar represents tumor samples and grey bar represents normal samples. (D) POLR1B was upregulated in NSCLC samples compared to normal tissues by analyzing GSE18842.(E-G) The expression of POLR1B was positively associated with (E) KRAS, (F) EGFR and (G) TP53 gene in NSCLC samples. ${ }^{*} \mathrm{P}<0.05,{ }^{* * * *} \mathrm{P}<0.001$ vs. normal tissues. POLR1B, polymerase 1 subunit $\mathrm{B}$; NSCLC, non-small cell lung cancer; LUAD, lung adenocarcinoma; LUSC, lung squamous cell carcinoma; KRAS, Kras; EGFR, epidermal growth factor receptor; TP53, tumor protein 53.

The present study focused on the functions and expression levels of POLR1B in NSCLC. GEPIA dataset analysis revealed that the POLR1B expression levels in LUAD (median expression level, 8.69) and LUSC (median expression level, 10.79) were higher than those in normal tissue samples (LUAD median expression level, 4.78; LUSC median expression level, 4.6) (Fig. 1C). Furthermore, the present study analyzed a public dataset, GSE18842, to further validate the GEPIA analysis. The results revealed that POLR1B was also upregulated in NSCLC samples compared with normal lung tissues (Fig. 1D). Furthermore, the association between POLR1B and several key NSCLC regulators, including the GTPases KRas (KRAS), epidermal growth factor receptor (EGFR), and tumor protein
(TP)53, was also assessed (Fig. 1E-G). The results suggested that the expression of POLR1B was positively associated with that of TP53, EGFR, KRAS and NRAS in NSCLC samples.

mRNA expression levels of POLRIB in lung cancer cell lines. RT-qPCR was performed in order to evaluate the expression levels of POLR1B in lung cancer cell lines, including A549, NCI-H1299, NCI-H1975 and NCI-H460 cells. The results revealed that the POLR1B mRNA expression levels in all four lung cancer cell lines were higher than those of the GAPDH control (Fig. 2A). Therefore, the data revealed that POLR1B was highly expressed in NSCLC cells, indicating that it may serve an important role in the regulation of lung cancer cells. 
POLRIB is effectively silenced by lentivirus-mediated RNA $i$ in A549 cells. Due to the high expression levels of POLR1B in lung cancer cell lines, lentivirus-mediated RNAi was used to suppress POLR1B expression in A549 cells to further investigate the functions of POLR1B. The mRNA expression levels were detected via RT-qPCR. The results revealed that the POLR1B mRNA expression levels were markedly decreased in POLR1B shRNA-silenced A549 cells, compared with the control group (Fig. 2B). In addition, western blotting was used to determine the POLR1B protein expression levels in A549 cells, and also revealed a significant decrease in these levels in POLR1B shRNA-lentivirus-infected cells (compared with cells infected with the negative control), reconfirming the results of mRNA detection (Fig. 2C). Collectively, these data revealed that the expression of POLR1B was effectively silenced in POLR1B shRNA-lentivirus infected A549 cells.

POLRIB knockdown inhibits the proliferation of lung cancer cells. In order to investigate whether POLR1B affected the proliferation of lung cancer cells, the Celigo ${ }^{\circledR}$ cell counting system was used to directly visualize and enumerate the proliferative rate of A549 cells. At the 5-day post-infection point, the green florescence signals of the shPOLR1B-lentivirus infected A549 cells were notably weaker than those of the control cells $(\mathrm{P}<0.05)$. In addition, the calculated cell counting results also suggested that POLR1B-silencing using an shPOLR1B-lentivirus decreased the proliferation rate of A549 cells (Fig. 3A).

Furthermore, an MTT assay was also used to investigate the proliferation rates of A549 cells following POLR1B-knockdown. According to the results, the proliferation rates of POLR1B-silenced A549 cells at 1, 2, 3, 4 and 5 days post-infection were markedly inhibited compared with those of the control cells (Fig. 3B).

In addition, a colony formation assay was used to assess the effects of POLR1B-silencing on the colony-forming ability of A549 cells. Compared with the control cells, the number of colonies in the POLR1B-silenced groups (as determine by Giemsa staining) was decreased (Fig. 3C). Quantitative analysis also revealed that the colonies of the POLR1B-silenced group possessed significantly fewer cells than those of the control-infected group (Fig. 3D).

In conclusion, these results demonstrated that POLR1B-knockdown inhibited the proliferation of A549 cells, indicating that POLR1B serves an important role in the regulation of lung cancer cell proliferation.

POLRIB suppression induces apoptosis in A549 cells. Based on the aforementioned findings, demonstrating that POLR1B serves an important role in the modulation of lung cancer cell proliferation, flow cytometry was performed to analyze apoptosis in A549 cells following POLR1B knockdown. The results revealed that the proportion of apoptotic cells was increased in the POLR1B-knockdown A549 cells (Fig. 4A). Quantitative analysis also demonstrated that the percentage of apoptotic cells infected with shPOLR1B lentivirus was over two times that of the control cells (Fig. 4B), indicating that POLR1B depletion induces lung cancer cell apoptosis.
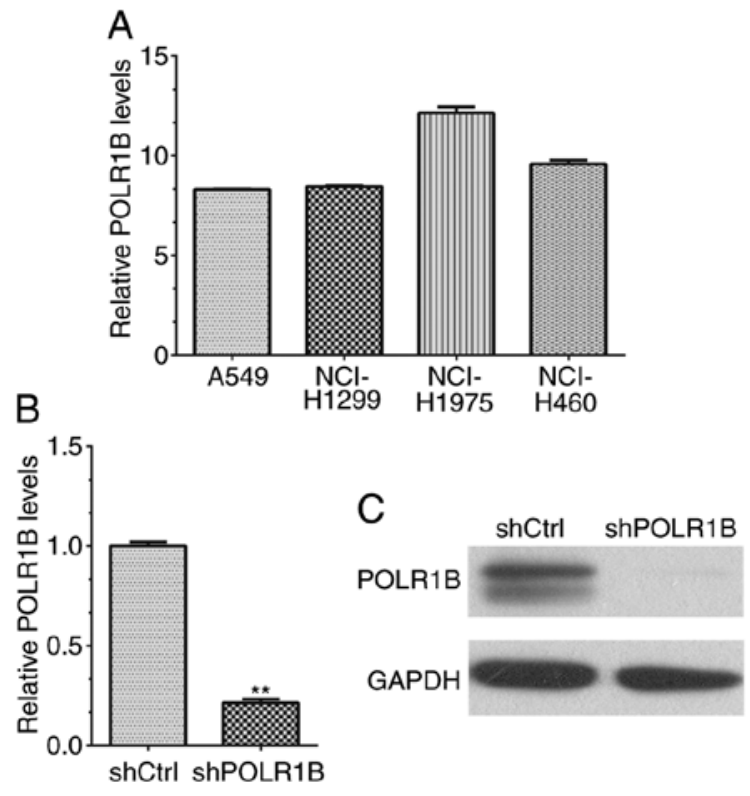

Figure 2. Silencing of POLR1B expressed by lentivirus mediated RNAi. (A) POLR1B mRNA levels in A549, NCI-H1299, NCI-H1975 and NCI-H460 lung cancer cell lines. (B) The knockdown efficiency of POLR1B determined by reverse transcription-quantitative PCR assay $(n=3)$. (C) The knockdown efficiency of POLR1B determined by western-blot assay. Values are presented as the mean \pm standard error of the mean. ${ }^{* *} \mathrm{P}<0.01$ vs. negative control group. POLR1B, polymerase 1 subunit B; RNAi, RNA interference; sh, short hairpin.
A

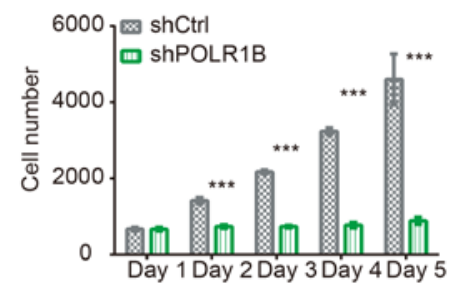

C

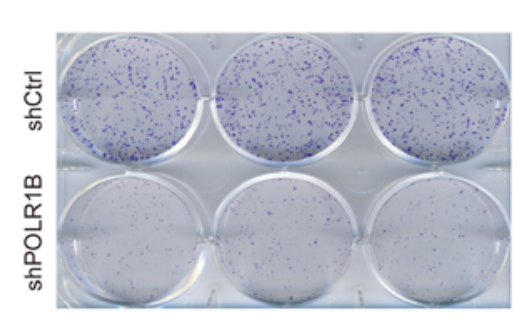

B
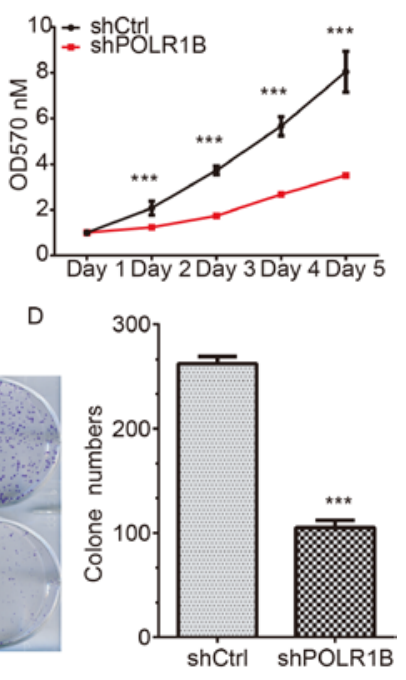

Figure 3. POLR1B knockdown suppresses the proliferation of A549 cells. (A) Celigo Cell Counting application assay demonstrated that POLR1B knockdown significantly suppressed A549 cell proliferation. (B) The proliferation rates of A549 cells infected with shCtrl lentivirus and shPOLR1B lentivirus were detected by MTT assay.(C) Cell colonies of A549 cells infected with shCtrl lentivirus and shPOLR1B lentivirus were stained with Giemsa. (D) Quantitative analysis of the number of A549 cell colonies infected with shPOLR1B lentivirus compared with shCtrl lentivirus. All experiments were performed independently at least three times. ${ }^{* * *} \mathrm{P}<0.001$ vs. negative control group. POLR1B, polymerase 1 subunit B; sh, short hairpin.

Identification of POLRIB targets using microarray co-expression analysis. In the present study, a microarray analysis was performed to identify the downstream targets of POLR1B. A total of 970 genes were identified as potential 

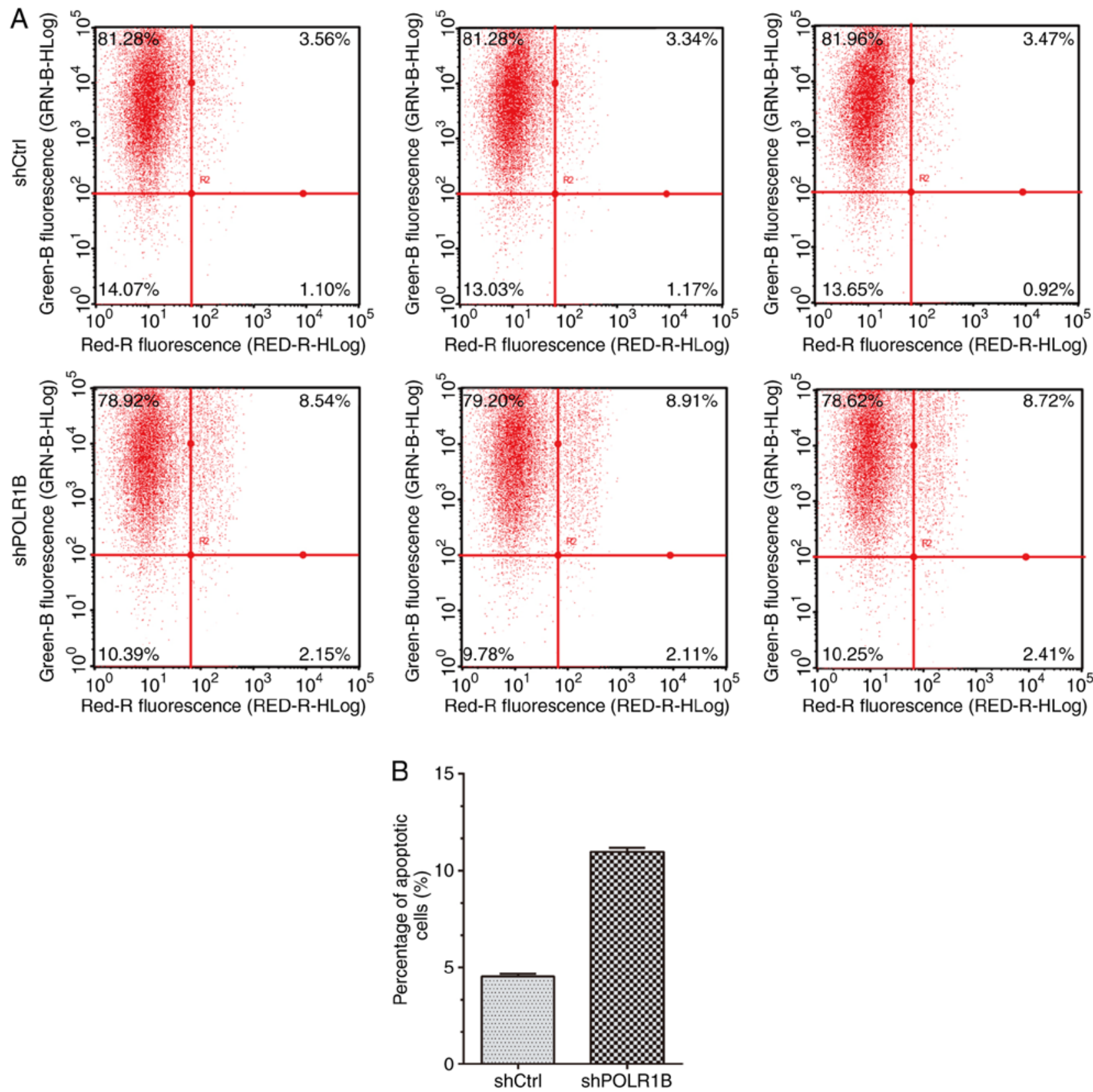

Figure 4. Effects of POLR1B depletion on A549 cells apoptosis. (A) The apoptosis rate of A549 cells infected with shCtrl lentivirus and shPOLR1B lentivirus was detected by flow cytometry. (B) Statistical analysis of the percentage of apoptotic cells. All experiments were performed independently at least three times. POLR1B, polymerase 1 subunit $\mathrm{B}$; sh, short hairpin.

targets of POLR1B, including 424 genes that were upregulated, and 546 that were downregulated following POLR1B knockdown in A549 cells (Fig. 5A). Co-expression analysis of POLR1B in NSCLC was then performed using the cBioPortal database, and POLR1B-target pairs with $\mathrm{P}<0.05$ were considered to be reliable. Combining the results of the microarray and co-expression analyses, 138 upregulated (Fig. 5B) and 164 downregulated (Fig. 5C) genes we identified as potential downstream targets of POLR1B. The top 10 upregulated and downregulated genes after POLR1B knockdown in A549 cells are listed Table I.

The bioinformatics analysis also demonstrated that POLR1B was involved in 'positive regulation of glucose import', 'autophagosome assembly,' 'fat cell differentiation', 'negative regulation of gene expression', 'positive regulation of cellular senescence', 'cellular amino acid biosynthetic process', 'keratan sulfate biosynthetic process', 'tetrahydrofolate metabolic process', 'liver regeneration' and 'negative regulation of transcription' (Fig. 5D).

Identification of key POLRIB targets in NSCLC using protein-protein interaction (PPI) network analysis. The key targets of POLR1B in NSCLC were identified using a PPI network analysis. The search tool for the retrieval of interacting genes/proteins (STRING) database (https://string-db.org) was used to investigate the interactions between POLR1B targets. As presented in Fig. 6, the upregulated target-mediated PPI network included 71 nodes and 71 edges, and the downregulated target-mediated PPI network possessed 128 nodes and 300 edges. Among these genes, adrenoceptor $\alpha 1 \mathrm{D}$ (ADRA1D; degree=5) 

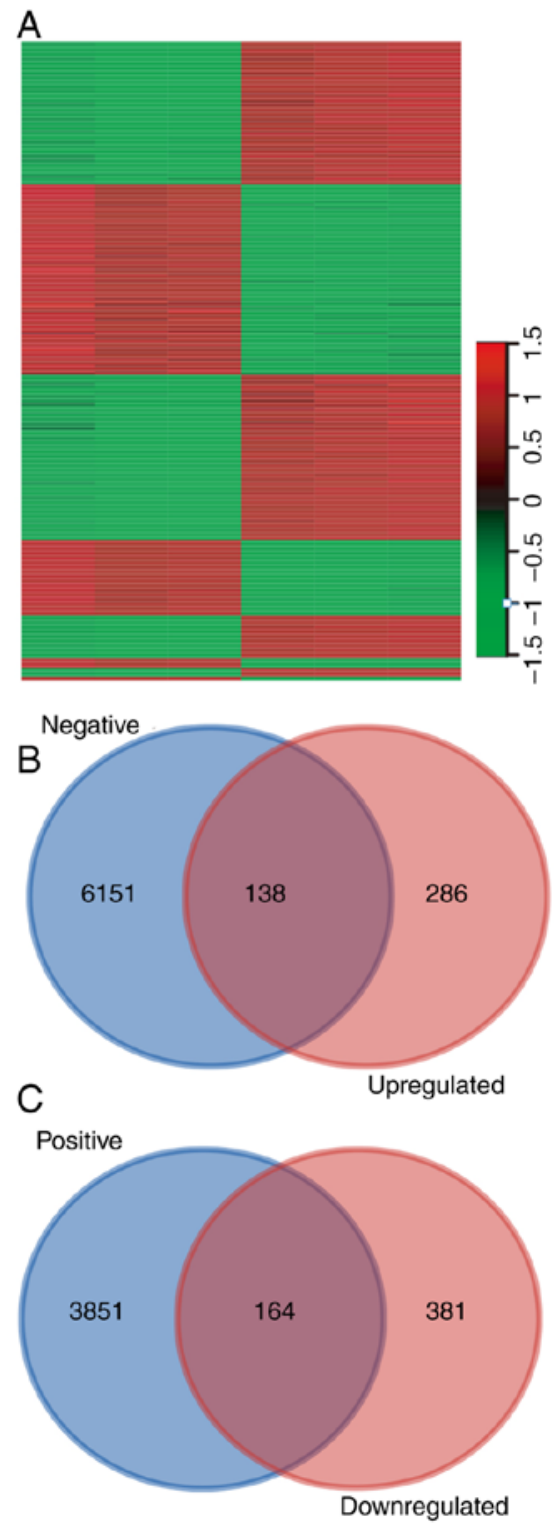

D

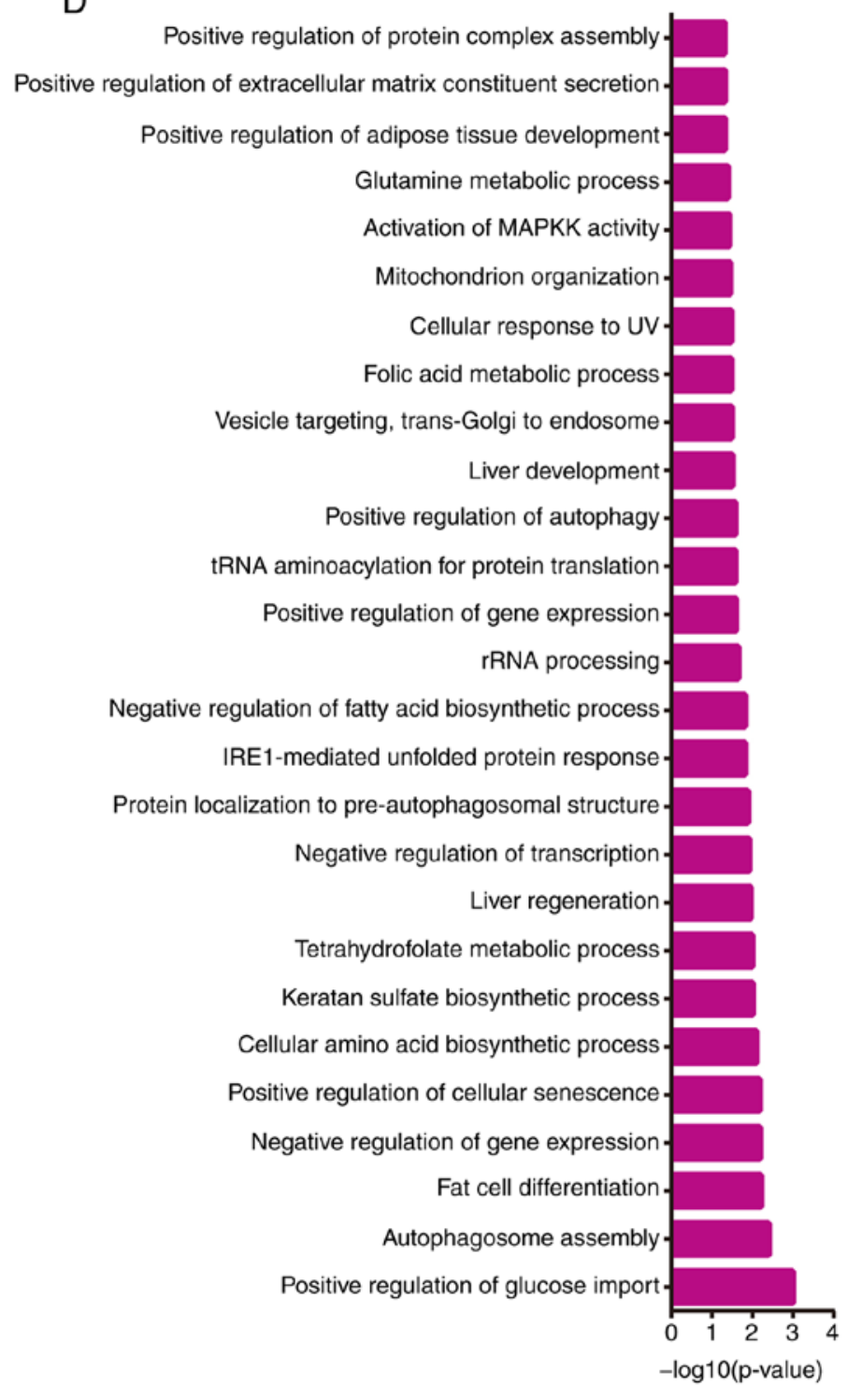

Figure 5. Identification of POLR1B targets in NSCLC. (A) Heatmap presenting the differently expressed genes in A549 following POLR1B knockdown. Green represents downregulated genes; red represents upregulated genes. (B) Venn map analysis of POLR1B targets in NSCLC by using microarray analysis and co-expression analysis. (C) Bioinformatics analysis of POLR1B functions in NSCLC using the Database for Annotation, Visualization and Integrated Discovery system. POLR1B, polymerase 1 subunit B; NSCLC, non-small cell lung cancer.

and nuclear receptor subfamily 4 group A member 1 (NR4A1; degree $=5$ ) in the upregulated PPI network, myc proto-oncogene protein $(\mathrm{MYC})($ degree $=5)$, ribosome biogenesis protein $\mathrm{BOP} 1$ (BOP1; degree=14), H/ACA ribonucleoprotein complex subunit DKC1 (DKC1; degree=13), RRP12-like protein (RRP12; degree=12), importin-4 (IPO4; degree=11), bifunctional methylenetetrahydrofolate dehydrogenase/cyclohydrolase, mitochondrial (MTHFD2; degree=11), CTP synthase 1 (CTPS1; degree $=9$ ), Glycine-tRNA ligase (GARS; degree $=8$ ), and nucleolar complex protein 2 homolog (NOC2L; degree $=7$ ) in the downregulated network were identified to be key regulators of NSCLC by interacting with POLR1B.

\section{Discussion}

Ribosome synthesis is one of the most complex and energy-demanding cellular processes, and the production of
rRNA, the first event in ribosome synthesis, serves a critical role in the complex regulation of cell proliferation (27). Enzymes involved in rRNA synthesis include RNA polymerase I (Pol I), which transcribes ribosomal DNA (rDNA) to generate a pre-rRNA precursor that is further processed into $18 \mathrm{~S}, 5.8 \mathrm{~S}$ and $28 \mathrm{~S}$ rRNA, which occurs via a number of essential and critical nucleolytic steps (28). Previous studies have suggested that increased Pol I-mediated rRNA synthesis in the nucleolus may be associated with poor prognosis in patients with rhabdomyosarcoma (19). Furthermore, POLR1B has been identified as one of the subunits of Pol I in human cells (15). Therefore, it was hypothesized that POLR1B may be involved in the regulation of tumor cells, particularly in lung cancer cell proliferation in the present study. However, the molecular functions of POLR1B in human cancer remain unknown. To the best of our knowledge, the present study was the first to demonstrate that POLR1B was highly expressed in 
Table I. Top 10 up- and downregulated genes following POLR1B knockdown in A549 cells.

\begin{tabular}{lrrrrrrrrrr}
\hline Gene & NC & \multicolumn{1}{c}{ NC } & \multicolumn{1}{c}{ NC } & \multicolumn{1}{c}{ KD } & KD & KD & FC & Regulation & P-value & FDR \\
\hline EREG & 10.08 & 10.04 & 10.11 & 6.65 & 6.72 & 6.77 & -10.30 & Down & $<0.001$ & 0.00 \\
STC2 & 8.76 & 8.74 & 8.77 & 5.58 & 5.71 & 5.56 & -8.83 & Down & $<0.001$ & 0.00 \\
RAB39B & 6.59 & 6.50 & 6.27 & 3.47 & 3.33 & 3.70 & -7.74 & Down & $<0.001$ & 0.00 \\
PCK2 & 8.35 & 8.50 & 8.31 & 5.87 & 5.25 & 5.66 & -6.93 & Down & $<0.001$ & 0.00 \\
IL11 & 7.22 & 7.29 & 6.92 & 4.23 & 4.48 & 4.48 & -6.70 & Down & $<0.001$ & 0.00 \\
ULBP1 & 6.29 & 6.34 & 6.25 & 3.21 & 4.11 & 3.40 & -6.57 & Down & $<0.001$ & 0.00 \\
TRIB3 & 10.47 & 10.47 & 10.45 & 7.73 & 7.75 & 7.82 & -6.48 & Down & $<0.001$ & 0.00 \\
H1F0 & 10.06 & 10.10 & 10.11 & 7.52 & 7.69 & 7.45 & -5.80 & Down & $<0.001$ & 0.00 \\
BCAT1 & 6.93 & 6.89 & 7.00 & 4.16 & 4.46 & 4.62 & -5.77 & Down & $<0.001$ & 0.00 \\
DDIT3 & 8.39 & 8.39 & 8.33 & 5.98 & 5.96 & 5.82 & -5.47 & Down & $<0.001$ & 0.00 \\
IFIT1 & 5.23 & 5.21 & 5.00 & 7.64 & 7.53 & 7.52 & 5.34 & Up & $<0.001$ & 0.00 \\
VTCN1 & 3.98 & 4.19 & 3.73 & 6.27 & 6.57 & 6.41 & 5.45 & Up & $<0.001$ & 0.00 \\
TPM1 & 7.48 & 7.57 & 7.43 & 10.05 & 10.17 & 10.12 & 6.15 & Up & $<0.001$ & 0.00 \\
GRAMD3 & 4.80 & 5.00 & 4.56 & 7.47 & 7.56 & 7.42 & 6.48 & Up & $<0.001$ & 0.00 \\
NANOS1 & 5.37 & 5.23 & 5.41 & 8.00 & 8.07 & 8.04 & 6.50 & Up & $<0.001$ & 0.00 \\
HSPA1A & 6.37 & 6.48 & 6.24 & 9.15 & 9.16 & 9.16 & 6.92 & Up & $<0.001$ & 0.00 \\
HSPA1B & 6.37 & 6.48 & 6.24 & 9.15 & 9.16 & 9.16 & 6.92 & Up & $<0.001$ & 0.00 \\
IFITM10 & 4.67 & 4.82 & 5.00 & 7.48 & 7.71 & 7.72 & 6.99 & Up & $<0.001$ & 0.00 \\
TPM2 & 4.66 & 4.84 & 4.29 & 7.46 & 7.56 & 7.52 & 7.56 & Up & $<0.001$ & 0.00 \\
RAP1A & 6.96 & 7.01 & 6.76 & 12.49 & 12.57 & 12.48 & 48.60 & Up & $<0.001$ & 0.00 \\
\hline
\end{tabular}

POLR1B, polymerase 1 subunit B; NC, shRNA control; KD, shPOLR1B; FDR, false discovery rate.

numerous lung cancer cell lines, including A549, NCI-H1299, NCI-H1975 and NCI-H460, which suggested that POLR1B plays a critical role in the regulation of lung cancer cells.

The essential components of Pol I include nucleolar transcription factor 1, RNA polymerase I-specific transcription initiation factor RRN3 (also known as TIF-1A) and one of the largest subunits, POLR1B, which have all been reported to be highly expressed in various types of cancer, including B-cell lymphoma (29). In addition, small molecule inhibitors that directly target Pol I (such as CX-5461 and CX-3543) have been developed and implemented in further clinical trials for the treatment of cancer $(30,31)$. Specifically, a previous study demonstrated that BMH-21, a compound that promotes proteasome-dependent destruction of the large catalytic subunit of the Pol I holocomplex (RPA194), possesses broad anti-tumorigenic activity across the NCI60 cancer cell lines, and suppresses tumor growth in vivo (32). Therefore, inhibiting the expression of POLR1B may also attenuate tumor development. The present study utilized several methods to assess cellular proliferation, including the Celigo ${ }^{\circledR}$ cell counting method, MTT and colony formation assays, and, to the best of our knowledge, demonstrated for the first time that lentivirus-mediated RNAi POLR1B-silencing inhibited the proliferation of lung cancer cells. These data suggest that POLR1B serves an important role in the modulation of lung cell proliferation. Furthermore, apoptosis was detected using flow cytometry following POLR1B-silencing in the present study, which revealed that POLR1B-knockdown induced apoptosis in A549 cells. Therefore, the present study suggests that suppressing POLR1B not only inhibits the proliferation of lung cancer cells, but also results in cancer cell apoptosis, indicating a double effect of POLR1B-depletion in cancer cells.

In order to investigate the molecular mechanisms underlying the involvement of POLR1B in NSCLC progression, microarray and co-expression analyses were performed. To the best of our knowledge, the results of the present study demonstrated for the first time that in NSCLC, POLR1B was involved in regulating multiple biological processes, including 'positive regulation of glucose import', 'autophagosome assembly', 'positive regulation of cellular senescence' and 'cellular amino acid biosynthetic process.' By constructing PPI networks, several key targets of POLR1B were also identified, including ADRA1D, NR4A1, MYC, BOP1, DKC1, RRP12, IPO4, MTHFD2, CTPS1, GARS and NOC2L. Previous studies also confirmed that these genes served prominent roles in NSCLC progression; NR4A1 was observed to be upregulated in NSCLC, and to promote tumor growth and metastasis. In addition, MYC was reported to be an oncogene in NSCLC, and to be involved in regulating cellular proliferation, the cell cycle, apoptosis and the immune response.

In conclusion, the present study identified that POLR1B may serve an important role in the regulation of lung cancer cell proliferation. Furthermore, POLR1B depletion was revealed to inhibit lung cancer cell proliferation and induce apoptosis. The suggestion that POLR1B functions as an important regulator of lung cancer may facilitate the development of effective therapeutic strategies for NSCLC. 
A

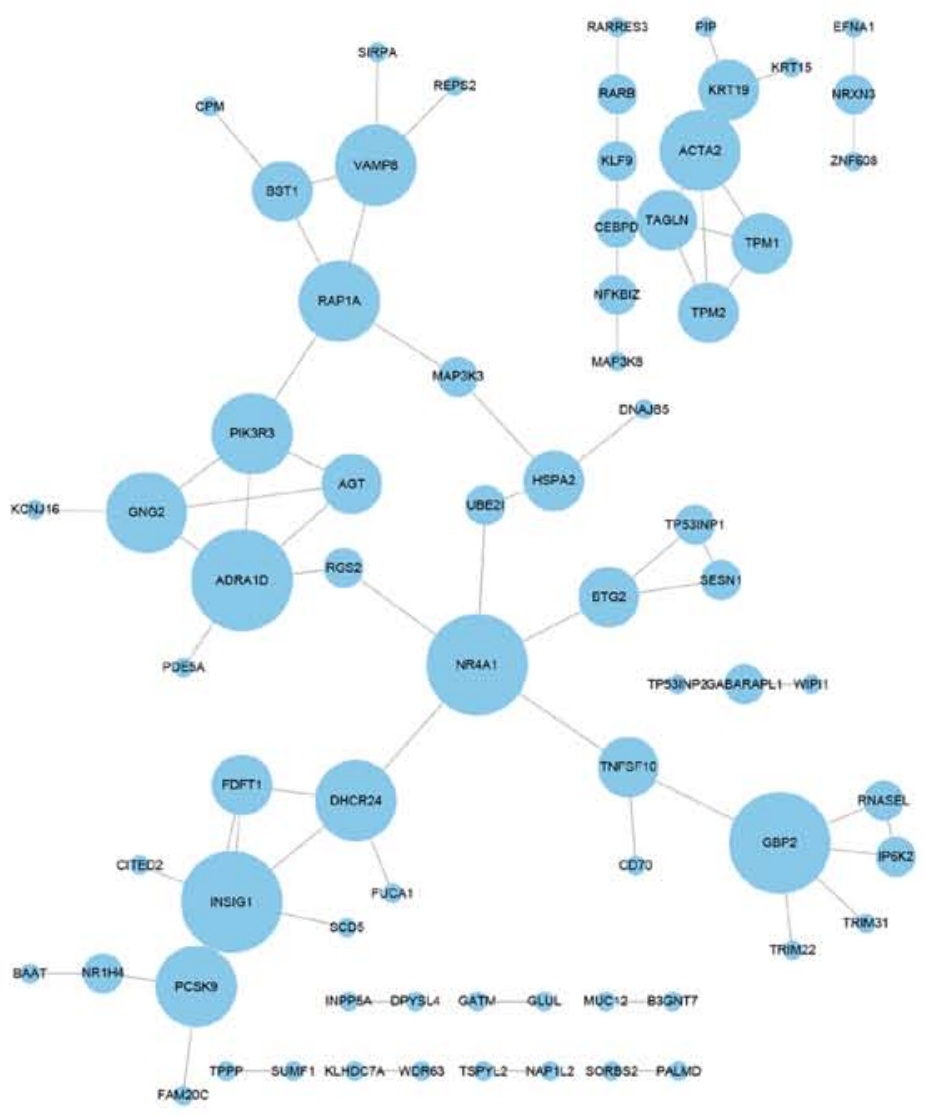

B

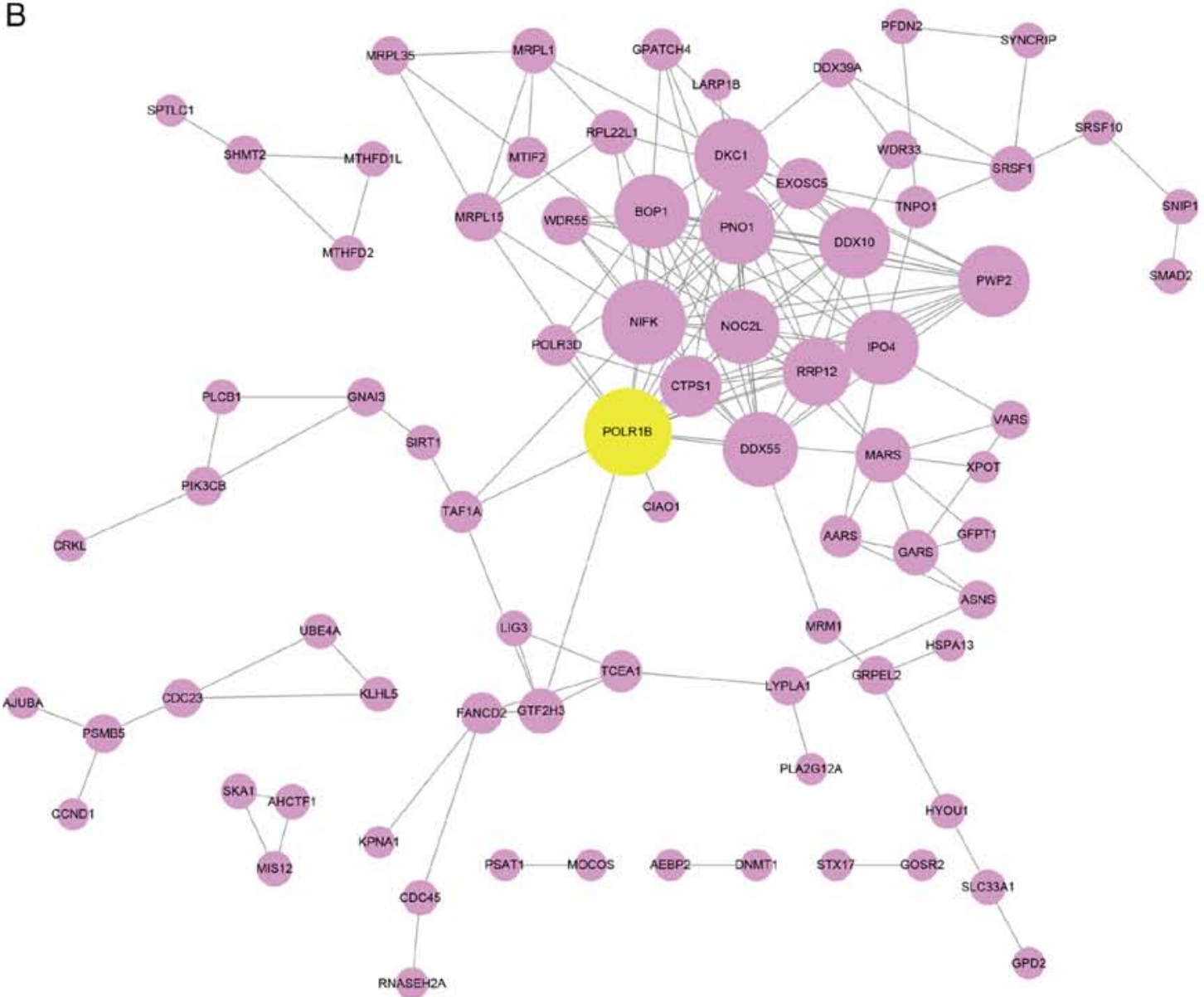

Figure 6. Construction of POLR1B-mediated PPI network in NSCLC. (A) PPI network revealing the associations between upregulated genes and (B) downregulated genes following POLR1B knockdown in A549 cells. POLR1B, polymerase 1 subunit B; PPI, protein-protein interaction; NSCLC, non-small cell lung cancer. 


\section{Acknowledgements}

Not applicable.

\section{Funding}

The present study was supported by grants from the Jiaxing Science and Technology Program (grant no. 2017AY33007), Zhejiang Province Medical and Health Technology Program (grant no. 2018KY800) and Jiaxing Key Discipiline of Medicine-Thoracic Surgery supporting project (grant no. 2019-zc-09).

\section{Availability of data and materials}

The datasets used and/or analyzed during the current study are available from the corresponding author on reasonable request.

\section{Authors' contributions}

FY and WQ designed the study and wrote the manuscript. FY, HL and JZ performed the experiments. XM analyzed the data. All authors read and approved the final version of the manuscript.

\section{Ethics approval and consent to participate}

Not applicable.

\section{Patient consent for publication}

Not applicable.

\section{Competing interests}

The authors declare that they have no competing interests.

\section{References}

1. Jemal A, Bray F, Center MM, Ferlay J, Ward E and Forman D Global cancer statistics. CA Cancer J Clin 61: 69-90, 2011.

2. Brahmer J, Reckamp KL, Baas P, Crinò L, Eberhardt et al: Nivolumab versus docetaxel in advanced squamous-cell non-small-cell lung cancer. N Engl J Med 373: 123-135, 2015.

3. Rosell R, Bivona TG and Karachaliou N: Genetics and biomarkers in personalisation of lung cancer treatment. Lancet 382: 720-731, 2013.

4. Gerber DE and Schiller JH: Maintenance chemotherapy for advanced non-small-cell lung cancer: New life for an old idea. J Clin Oncol 31: 1009-1020, 2013.

5. Brudno JN and Kochenderfer JN: Chimeric antigen receptor T-cell therapies for lymphoma. Nat Rev Clin Oncol 15: 31-46, 2018.

6. Anagnostou V, Smith KN, Forde PM, Niknafs N, Bhattacharya R, White J, Zhang T, Adleff V, Phallen J, Wali N, et al: Evolution of neoantigen landscape during immune checkpoint blockade in non-small cell lung cancer. Cancer Discov 7: 264-276, 2017.

7. Rizvi NA, Hellmann MD, Snyder A, Kvistborg P, Makarov V, Havel JJ, Lee W, et al: Cancer immunology. Mutational landscape determines sensitivity to PD-1 blockade in non-small cell lung cancer. Science 348: 124-128, 2015.

8. Garon EB, Rizvi NA, Hui R, Leighl N, Balmanoukian AS Eder JP, Patnaik A, Aggarwal C, Gubens M, Horn L, et al: Pembrolizumab for the treatment of non-small-cell lung cancer. N Engl J Med 372: 2018-2028, 2015.

9. Erfani N, Mehrabadi SM, Ghayumi MA, Haghshenas MR, Mojtahedi Z, Ghaderi A and Amani D: Increase of regulatory $\mathrm{T}$ cells in metastatic stage and CTLA-4 over expression in lymphocytes of patients with non-small cell lung cancer (NSCLC). Lung Cancer 77: 306-311, 2012.
10. Sharpe AH and Pauken KE: The diverse functions of the PD1 inhibitory pathway. Nat Rev Immunol 18: 153-167, 2018.

11. Neelapu SS, Tummala S, Kebriaei P, Wierda W, Gutierrez C, Locke FL, Komanduri KV, Lin Y, Jain N, Daver N, et al: Chimeric antigen receptor T-cell therapy-assessment and management of toxicities. Nat Rev Clin Oncol 15: 47-62, 2018.

12. Ludin A and Zon LI: Cancer immunotherapy: The dark side of PD-1 receptor inhibition. Nature 552: 41-42, 2017.

13. Torreira E, Louro JA, Pazos I, González-Polo N, Gil-Carton D, Duran AG, Tosi S, Gallego O, Calvo O and Fernández-Tornero C: The dynamic assembly of distinct RNA polymerase I complexes modulates rDNA transcription. Elife 6: pii: e20832, 2017.

14. Engel C, Gubbey T, Neyer S, Sainsbury S, Oberthuer C, Baejen C, Bernecky C and Cramer P: Structural basis of RNA polymerase $\mathrm{i}$ transcription initiation. Cell 169: 120-131.e22, 2017.

15. Andersen JS, Lam YW, Leung AK, Ong SE, Lyon CE, Lamond AI and Mann M: Nucleolar proteome dynamics. Nature 433: 77-83, 2005.

16. Seither P and Grummt I: Molecular cloning of RPA2, the gene encoding the second largest subunit of mouse RNA polymerase I. Genomics 37: 135-139, 1996.

17. Chan JC, Hannan KM, Riddell K, Ng PY, Peck A, Lee RS, Hung S, Astle MV, Bywater M, Wall M, et al: AKT promotes rRNA synthesis and cooperates with c-MYC to stimulate ribosome biogenesis in cancer. Sci Signal 4: ra56, 2011.

18. Drygin D, Rice WG and Grummt I: The RNA polymerase I transcription machinery: An emerging target for the treatment of cancer. Annu Rev Pharmacol Toxicol 50: 131-156, 2010.

19. Williamson D, Lu YJ, Fang C, Pritchard-Jones K and Shipley J: Nascent pre-rRNA overexpression correlates with an adverse prognosis in alveolar rhabdomyosarcoma. Genes Chromosomes Cancer 45: 839-845, 2006.

20. Tang Z, Li C, Kang B, Gao G, Li C and Zhang Z: GEPIA: A web server for cancer and normal gene expression profiling and interactive analyses. Nucleic Acids Res 45: W98-W102, 2017.

21. Cerami E, Gao J, Dogrusoz U, Gross BE, Sumer SO, Aksoy BA, Jacobsen A, Byrne CJ, Heuer ML, Larsson E, et al: The cBio cancer genomics portal: An open platform for exploring multidimensional cancer genomics data. Cancer Discov 2: 401-404, 2012.

22. Sanchez-Palencia A, Gomez-Morales M, Gomez-Capilla JA, Pedraza V, Boyero L, Rosell R and Fárez-Vidal ME: Gene expression profiling reveals novel biomarkers in nonsmall cell lung cancer. Int J Cancer 129: 355-364, 2011.

23. Nabzdyk CS, Chun M, Pradhan L and Logerfo FW: High throughput RNAi assay optimization using adherent cell cytometry. J Transl Med 9: 48, 2011.

24. Livak KJ and Schmittgen TD: Analysis of relative gene expression data using real-time quantitative PCR and the 2(-Delta Delta C(T)) method. Methods 25: 402-408, 2001.

25. Szklarczyk D, Franceschini A, Wyder S, Forslund K, Heller D, Huerta-Cepas J, Simonovic M, Roth A, Santos A, Tsafou KP, et al: STRING v10: Protein-protein interaction networks, integrated over the tree of life. Nucleic Acids Res 43 (Database Issue): D447-D452, 2015.

26. Su G, Morris JH, Demchak B and Bader GD: Biological network exploration with Cytoscape 3. Curr Protoc Bioinformatics 47: 8 13 11-24, 2014.

27. White RJ: RNA polymerases I and III, non-coding RNAs and cancer. Trends Genet 24: 622-629, 2008.

28. Cornelison R, Dobbin ZC, Katre AA, Jeong DH, Zhang Y, Chen D, Petrova Y, Llaneza DC, Steg AD, Parsons L, et al: Targeting RNA-polymerase I in both chemosensitive and chemoresistant populations in epithelial ovarian cancer. Clin Cancer Res 23: 6529-6540, 2017.

29. Bywater MJ, Poortinga G, Sanij E, Hein N, Peck A, Cullinane C, Wall M, Cluse L, Drygin D, Anderes K, et al: Inhibition of RNA polymerase I as a therapeutic strategy to promote cancer-specific activation of p53. Cancer Cell 22: 51-65, 2012.

30. Drygin D, Siddiqui-Jain A, O'Brien S, Schwaebe M, Lin A, Bliesath J, Ho CB, Proffitt C, Trent K, Whitten JP, et al: Anticancer activity of CX-3543: A direct inhibitor of rRNA biogenesis. Cancer Res 69: 7653-7661, 2009.

31. Ruggero D: Revisiting the nucleolus: From marker to dynamic integrator of cancer signaling. Sci Signal 5: pe38, 2012.

32. Peltonen K, Colis L, Liu H, Trivedi R, Moubarek MS, Moore HM, Bai B, Rudek MA, Bieberich CJ and Laiho M: A targeting modality for destruction of RNA polymerase I that possesses anticancer activity. Cancer Cell 25: 77-90, 2014.

This work is licensed under a Creative Commons Attribution-NonCommercial-NoDerivatives 4.0 International (CC BY-NC-ND 4.0) License. 